http://dx.doi.org/10.21611/qirt.1994.038

\title{
Analytical simulation of a multi-dimensional temperature field produced by planar defects of any shape; application to non-destructive testing
}

\author{
by DEGIOVANNI A., BENDADA A., BATSALE J.C. and MAILLET D. *
}

" Laboratoire d'Energétique et de Mécanique Théorique et Appliquée, URA CNRS 875, Institut National Polytechnique de Lorraine et Université de Nancy I, ENSEM, 2 avenue de la Forêt de Haye, 54500 Vandoeuvre-Les-Nancy, France.

\begin{abstract}
Thermal interface resistances are associated with bad contact between two materials. In the case where this interface takes place between two rectangular slabs of the same material, a heat pulse experiment with recording of the slab surface temperature evolution, gives information about the space distribution of this resistance. The inverse problem is considered here, using an explicit analytical solution of the direct problem and a stochastic approach, in the case of a non uniform interface resistance distribution. Experimental inversion of frames produced by an infrared camera is implemented.
\end{abstract}

\section{Nomenclature}

a diffusivity

a, b vectors

A, B quadrupole matrices

C, D quadrupole matrices

$e$ thickness of the slab

$e_{1, e 2}$ thickness of the two layers on each side of the defect

$\ell \quad$ width of the slab

$\mathrm{m}$ number of time steps

$\mathrm{N}$ number of space steps

$n$ number of harmonics considered

N convolution matrix

p Laplace parameter

$Q$ - absorbed energy by unit area

$R \quad$ interface thermal resistance

$T$ temperature $(=0$ at $t=0)$

$t$ time

$u$ coefficient

$x \quad$ direction parallel to the faces of the slab

$z$ direction normal to the faces of the slab

\section{Greek symbols}

$\alpha \quad$ eigenvalue

$\rho c, \lambda$ volumetric heat and conductivity

$\sigma \quad$ standard deviation of noise on $\Delta T^{*}$

$\tau \quad$ Laplace temperature

$\boldsymbol{\theta}$ Laplace-Fourier temperature

$\varphi \quad$ heat flux density

$\psi \quad$ Laplace flux density

$\phi \quad$ Laplace-Fourier flux

$\rho, \rho$ Fourier transform of $R(x)$ and spectrum

Superscripts or subscripts

- reduced quantity (dimensionless)

inf under resistance plane

sup above resistance plane

$a, \rho$ relative to $a$ and $\rho$

$i$ relative to eigenvalue number $i$

- relative to sane slab

$x, z \quad$ in $x$-and $z$-directions

1,2 relative to each layer 1 or 2

\section{Introduction}

Interface thermal resistances are the consequence of a structural discontinuity between two materials. Examples can be found in composite laminates (carbon fiber reinforced polymers): a delamination between two plies is characterized by an air gap that may affect the mechanical behaviour of the structure under load conditions; This delamination also creates an additional resistance to heat flow if the laminate is thermally stimulated. Such is also the case in welding applications where one looks for a perfect bonding between two materials.

A sketch of the interface thermal resistance between two slabs is shown in figure 1 in a twodimensional case. The problem that is considered in any non destructive evaluation technique is 
http://dx.doi.org/10.21611/qirt.1994.038

the detection of this type of defect and a quantification of its location, extent ( $x$-direction) and magnitude.

Cases where this resistance is a delamination between two plies in stratified composites have been considered in earlier works [1, 2]: a photothermal stimulation of the front face of the slab with the simultaneous temperature recording of the thermal field on either side of it allowed an estimation of both depth and resistance of the delamination in cases where this resistance was uniform in space. A one-dimensional transient model was used for inversion, after local normalization of the recorded infrared signal. The present work is the continuation of [3 and 4] where two- and thre-dimensional effects were taken into account but we consider now that the interface resistance is a space-function $R(x)$.

\section{Two-dimensional model}

The geometry of the problem is shown in figure 1 (cross-section $x-z$ of the slab). The slab (dimensions: $\ell \times e$ ) is made out of an homogeneous anisotropic material, one of its principal axes of anisotropy corresponding to the front face axis (conductivities $\lambda_{x}$ and $\lambda_{z}$, volumetric heat $\rho c$ ). The defect has been modeled by an interface resistance $R$, located at a constant depth $e_{1}$ beneath the front side. It is considered as a function of the $x$ coordinate. The Dirac heat flux density of the stimulation (absorbed energy density $Q$ ) is supposed to be uniform on front face, the initial slab temperature is assumed uniform $(T=0)$ and the lateral sides $(x=0$ and $x=\ell)$ and the front and rear faces $(z=0$ and $z=e)$ are insulated. Temperature $T$ obeys the following equation :

$$
\lambda_{\mathrm{x}} \frac{\partial^{2} T}{\partial x^{2}}+\lambda_{\mathrm{z}} \frac{\partial^{2} T}{\partial z^{2}}=\rho c \frac{\partial T}{\partial t}
$$

\subsection{Model in Laplace domain}

It is possible to apply a time Laplace transformation to equation (1) and to its associated boundary, interface $\left(z=e_{1}\right)$ and initial conditions. The Laplace temperature $\tau$ defined thereafter obeys the following system of equations:

$$
\begin{gathered}
\tau=\int_{0}^{\infty} \exp (-p t) T \mathrm{~d} t \\
\frac{\lambda_{\mathrm{x}}}{\lambda_{\mathrm{z}}} \frac{\partial^{2} \tau}{\partial x^{2}}+\frac{\partial^{2} \tau}{\partial z^{2}}-\frac{p}{a_{\mathrm{z}}} \tau=0 \\
\frac{\partial \tau}{\partial x}=0 \quad \text { for } x=0, \ell \quad \text { and: } \quad \frac{\partial \tau}{\partial z}=0 \quad \text { for } z=e \\
\frac{\partial \tau^{\text {sup }}}{\partial z}=\frac{\partial \tau^{\text {inf }}}{\partial z} \quad \text { and } \tau^{\text {sup }}-\tau^{\text {inf }}=R(x)\left[-\lambda_{z} \frac{\partial \tau}{\partial z}\right] \quad \text { for } z=e_{1}
\end{gathered}
$$

$a_{\mathrm{Z}}\left(=\lambda_{\mathrm{Z}} / \rho c\right)$ being the thermal diffusivity of the slab in the $z$ direction and superscripts $\sup$ and inf relating to conditions above or beneath the resistance plane (see figure 1). It is possible to make the axial ( $z$ direction) Laplace heat flux density $\Psi$ appear in boundary conditions relative to $z=0, e_{1}$ and $e$, that is the Laplace transfonn of the axial heat flux density $\varphi$ :

$$
\psi=\int_{0}^{\infty} \exp (-p t) \varphi d t \quad \text { with: } \quad \varphi=-\lambda_{z} \frac{\partial T}{\partial z}
$$

In order to reduce the number of parameters in the problem, it is very convenient to use dimensionless variables: 
http://dx.doi.org/10.21611/qirt.1994.038

$$
\begin{aligned}
t^{*} & =a_{\mathrm{z}} t / e^{2} ; p^{*}=e^{2} p / a_{\mathrm{z}} ; T^{*}=\rho c e T / Q ; \tau^{*}=\lambda_{\mathrm{z}} \tau /(Q e) \\
\varphi^{*} & =e^{2} \varphi /(a Q) ; \quad \psi^{*}=\psi / Q ; \quad x^{*}=\frac{x}{e}\left(\lambda_{\mathrm{z}} / \lambda_{\mathrm{x}}\right)^{1 / 2} ; z^{*}=z / e \\
\ell^{*} & =\frac{\ell}{e}\left(\lambda_{\mathrm{z}} / \lambda_{\mathrm{x}}\right)^{1 / 2} ; \quad R^{*}\left(x^{*}\right)=R(x) /\left(e / \lambda_{\mathrm{z}}\right) ; \quad e_{1}^{*}=e_{1} / e
\end{aligned}
$$

In the following part of this article, superscript * will be omitted for simplicity reasons.

\subsection{Model in Laplace-Fourier domain}

Using the space pulsations $\alpha$ that take the discrete values of the eigenvalues $\alpha=\alpha_{\mathrm{i}}=\mathrm{i} \pi / \ell$ (with $\mathrm{i}$ : natural integer)of the problem, the space cosine Fourier transform pair of function $\tau(x, z, p)$ is defined according to:

$$
\theta(\alpha, z, p)=\int_{0}^{\ell} \tau(x, z, p) \cos (\alpha x) d x ; \tau(x, z, p)=\frac{1}{\ell}\left[\theta_{0}+2 \sum_{i=1}^{\infty} \theta_{i} \cos \left(\alpha_{i} x\right)\right]
$$

with: $\theta_{i}=\theta\left(\alpha_{i}, z, p\right)$. Equation (3a) becomes then :

$$
\frac{\partial^{2} \theta}{\partial z^{2}}-\left(p+\alpha^{2}\right) \theta=0
$$

One can note that derivation of equation (6a) is made using the eigenvalues as particular values of the pulsations $\alpha_{i}$ as well as the $x$-boundary conditions.

If $\phi$ is the cosine Fourier transform of the reduced Laplace flux density $\Psi$, that is equal to unity for $z=0$ and to zero for $z=1$ (two faces of the slab), and that is the same on each side of the interface $(z=e 1)$, the $z$-boundary and interface conditions become:

$$
\begin{array}{ll}
\phi(\alpha, 0, p)=\frac{\sin (\alpha \ell)}{\alpha} & \text { for } z=0 \\
\theta^{\sup }-\theta^{\text {inf }}=\int_{0}^{\ell} R(x) \Psi(x, z, p) \cos (\alpha x) \mathrm{dx} & \text { for } z=e_{1} \\
\phi(\alpha, 1, p)=0 & \text { for } z=1
\end{array}
$$

If $\theta(0), \theta(1), \phi(0), \phi(1)$ are the column vectors having each $n+1$ components (subscripts $i=$ 0 to $n)$ that constitute the spectra $\theta_{i}(0$ or 1$)$ and $\phi_{i}(0$ or 1$)$ of Laplace temperature and flux density on the front or rear face, system (6) can be written using three matrices (or 2D quadrupoles, that is two-terminal-pair networks) that stem from integration of equation (6a) with respect to $z$ [5]. The first two of them are associated with layers (1) and (2), respectively above and beneath the interface plane of figure 1:

$$
\begin{aligned}
& {\left[\begin{array}{l}
\theta(0) \\
\phi(0)
\end{array}\right]=\left[\begin{array}{ll}
A_{1} & B_{1} \\
C_{1} & D_{1}
\end{array}\right]\left[\begin{array}{c}
\theta^{\text {sup }} \\
\phi\left(e_{1}\right)
\end{array}\right] \quad \text { with } \quad A_{q}=\left[\begin{array}{cccc}
A_{q}\left(\alpha_{0}\right) & 0 & . . & 0 \\
0 & A_{q}\left(\alpha_{1}\right) & . . & 0 \\
. . & 0 & . . & 0 \\
0 & . . & 0 & A_{q}\left(\alpha_{n}\right)
\end{array}\right]} \\
& {\left[\begin{array}{c}
\theta^{\text {inf }} \\
\phi\left(e_{1}\right)
\end{array}\right]=\left[\begin{array}{ll}
A_{2} & B_{2} \\
C_{2} & D_{2}
\end{array}\right]\left[\begin{array}{l}
\theta(1) \\
\phi(1)
\end{array}\right]}
\end{aligned}
$$

for $q=1$ or 2 . Diagonal matrices $B_{q}, C_{q}, D_{q}$ are constructed the same way: 
http://dx.doi.org/10.21611/qirt.1994.038

$$
\begin{aligned}
& A_{q}\left(\alpha_{\mathrm{i}}\right)=D_{q}\left(\alpha_{\mathrm{i}}\right)=\cosh \left(u_{\mathrm{i}} e_{q}\right) \quad B_{q}\left(\alpha_{\mathrm{i}}\right)=\frac{1}{u_{\mathrm{i}}} \sinh \left(u_{\mathrm{i}} e_{q}\right) \\
& C_{q}\left(\alpha_{\mathrm{i}}\right)=u_{\mathrm{i}} \sinh \left(u_{\mathrm{i}} e_{q}\right) \quad \text { with: } \quad u_{\mathrm{i}}=\left(p+\alpha_{\mathrm{i}}{ }^{2}\right)^{1 / 2} \text { for } \mathrm{i}=0 \text { to } \mathrm{n}
\end{aligned}
$$

In order to consider the complete spectra of Laplace temperatures and flux density, one should take $n=\infty$; In practice $n$ will be the number of harmonics that will be considered.The right member of equation (6c) is in fact the convolution product (noted * in Fourier space) between the Fourier cosine transform of $R(x)$ and the corresponding transform $\phi\left(e_{1}\right)$ of the Laplace flux $\Psi$ at the location of the interface:

and:

$$
\boldsymbol{\theta}^{\mathrm{m} \rho}-\boldsymbol{\theta}^{\mathrm{inf}}=\boldsymbol{\rho} * \boldsymbol{\phi}\left(e_{1}\right)=\mathbf{N}_{\boldsymbol{\rho}} \boldsymbol{\phi}\left(e_{1}\right) \quad \text { with: } \quad \boldsymbol{\rho}=\left[\begin{array}{llll}
\rho_{0} & \rho_{\mathrm{l}} & . . & \rho_{\mathrm{n}}
\end{array}\right]^{\mathrm{t}}
$$

$$
\mathbf{N}_{\rho}=\frac{1}{\ell}\left[\begin{array}{ccccc}
\rho_{0} & 2 \rho_{1} & 2 \rho_{2} & . . & 2 \rho_{n} \\
\rho_{1} & \rho_{0}+\rho_{2} & \rho_{1}+\rho_{3} & . . & \rho_{n-1}+\rho_{n+1} \\
\rho_{2} & \rho_{1}+\rho_{3} & \rho_{0}+\rho_{4} & . . & \rho_{n-2}+\rho_{n+2} \\
. & . . & . . & . . & . . \\
\rho_{n} & \rho_{n-1}+\rho_{n+1} & \rho_{n-2}+\rho_{n+2} & . . & \rho_{0}+\rho_{2 n}
\end{array}\right] \quad \text { and: } \rho_{i}=\int_{0}^{\ell} R(x) \cos \left(\alpha_{i} x\right) d x
$$

$\mathbf{N}_{\rho}$ is a convolution matrix constructed with $\boldsymbol{\rho}$, the column vector that contains all the components of the spectrum of $R(x)$. Finally heat transfer through the slab can be written the following way:

$$
\left[\begin{array}{l}
\theta(0) \\
\phi(0)
\end{array}\right]=\left[\begin{array}{ll}
A_{1} & B_{1} \\
C_{1} & D_{1}
\end{array}\right]\left[\begin{array}{cc}
I & N_{\rho} \\
0 & I
\end{array}\right]\left[\begin{array}{ll}
A_{2} & B_{2} \\
C_{2} & D_{2}
\end{array}\right]\left[\begin{array}{ll}
\theta(1) \\
\phi(1)
\end{array}\right]
$$

$I$ and 0 being respectively the identity matrix and the zero matrix of order $(n+1)$. Starting from this equation, it is possible to find the front or rear face thermal contrast in Laplace-Fourier (LF) domain, $\Delta \boldsymbol{\theta}(0$ or 1$)$, that is the double LF transform of the reduced temperature difference $\Delta T^{*}$ between a point located on the face of a defectuous slab (reduced temperature $T^{\star}=\rho$ c:e $T / Q$ ) and the corresponding point belonging to a sane slab (reduced temperature $T_{0}^{*}$ for $R(x)=0$ ):

$$
\begin{aligned}
& \Delta \theta(0)=C_{2} C^{-1} N_{\rho} C_{2}\left(C+C_{1} N_{\rho} C_{2}\right)^{-1} \phi(0) \\
& \Delta \theta(1)=-\left(C+C_{1} N_{\rho} C_{2}\right)^{-1} C_{1} N_{\rho} C_{2} C^{-1} \phi(0)
\end{aligned}
$$

with: $\mathbf{C}=\mathbf{C}_{1} \mathbf{A}_{2}+\mathbf{A}_{1} \mathbf{C}_{2} \quad ; \phi(0)=\left[\begin{array}{lllll}\ell & 0 & 0 & \ldots . . & 0\end{array}\right]^{2}$ and $\phi(1)=\mathbf{0}$

\section{The inverse problem}

\subsection{Explicit inversion}

The problem considered here is to estimate function $R(x)$ starting from measurement of $T(x, z=0, t)$. Two quadratures of the measured signal, corresponding to transformation (2), with the calculation of $\tau(x, z=0, p)$, and of the reduced Laplace contrast $\Delta \tau(x, z=0, p)$ - and to transformation (5), allow the estimation of vector $\Delta \boldsymbol{\theta}(z=0, p)$, $p$ being any value of the reducad Laplace variable. The number $(n+1)$ of components of $\Delta \theta$ must be smaller than the number of space points sampled (in $x$ ), in order to prevent aliasing (Shannon's theorem). $\Delta \boldsymbol{\theta}$ is now considered as the new input signal that will be used for inversion, instead of the original one $T(x, z=0, t)$. Equation (10a), for front face detection, can be put under the following form: 
http://dx.doi.org/10.21611/qirt.1994.038

$\mathbf{N}_{\rho} \mathbf{a}=\boldsymbol{\rho} * \mathbf{a}=\mathbf{b}$ with: $\mathbf{a}=\mathbf{C}_{2} \mathbf{C}^{-1} \phi(0)-\mathbf{C}_{1} \Delta \theta(0, p)$ and $\mathbf{b}=\mathbf{C C}_{2}^{-1} \Delta \theta(0, p)$

Because of the commutative property of the convolution product (11), this equation is oquivalent to:

$$
\mathbf{N}_{,} \mathbf{p}=\mathbf{b} \Rightarrow \boldsymbol{p}=\mathbf{N}^{-i} \mathbf{b} \Rightarrow R(x)=\frac{1}{\ell}\left[\rho_{0}+2 \sum_{i=1}^{n} \rho_{i} \cos \left(\alpha_{i} x\right)\right]
$$

where matrix $\mathrm{Na}_{a}$ is constructed starting from components of vector a the same way as $\mathbf{N}_{\mathbf{p}}$ starting from $\rho$ in its definition following equation (8). An alternate explicit inversion algorithm, using $A(x)$ and $B(x)$, the originals of vectors $a$ and $b$, can be found if one remarks that convolution product (11) in Fourier space corresponds to a simple product in the original space:

$$
R(x) A(x)=B(x) \Rightarrow R(x)=\frac{B(x)}{A(x)}
$$

\subsection{Simulation of inversion}

A simulation of inversion has been implemented for a "smooth" interface resistance function (reduced depth: $e_{1}=0.25$ - reduced width of the slab: $\ell=10$ ), whose spectrum is given in figure 2a; $R(x)$ is the sum of two functions proportional to $\left[1+\left(x-x_{1} \text { or } 2\right)^{2}\right]^{-1}, x_{1}$ and $x_{2}$ being the locations of the two local maxima. This function is plotted in figure $2 b$ (curve 1 ) : it is a "camel type". function with two humps and is used to test the separative power of the inversion procedure. One can notice that $n=10$ harmonics are enough to characterize this function. The direct model, based on equation (10a), has produced a front face contrast field $\Delta T(t, x)$, numerical Laplace inversion being done via Stehfest algorithm (see [4]). A contrast thermogram $\Delta T(t)$ corresponding to the top of the lower hump of figure $2 b$ is plotted in figure $2 c(N=128$ points for Fourier space quadrature, $m=100$ for Laplace time quadrature). A computer generated random noise of standard deviation $\sigma=0.1$ (which is huge compared to the maximum level of contrast at that point: signal over noise ratio of 2 ) has been added to this contrast and is also plotted in figure 2c. Estimated profiles obtained with a time step $\Delta t=0.01$ and $p=1$ (curve 2) or $p$ $=5$ (curve 3 ) are plotted in figure $2 b$. They correspond to the inversion based on equation (13); Use 0 " equations (12) leads to nearly the same profile with however larger oscillations. The agreement seems to be very good between exact and estimated functions.

A similar procedure has been implemented for a piecewise constant resistance: a "double door" that is plotted in figure $3 a\left(e_{1}=0.16, \ell=9.235\right)$. It corresponds to a square isotropic sample whose dimensional characteristics are the following: $\ell=58 \mathrm{~mm}, e=6.28 \mathrm{~mm}, e_{1}=1 \mathrm{~mm}$. The estimated profiles using the same level of noise $(\sigma=0.1)$ as before with $n=40$ harmonics (curve 2) and $n=30$ harmonics (curve 3 ) - $\Delta t=0.01, m=100, N=128, p=9$ - are plotted in the same figure $3 a$; One can notice that the results are not as good as in the preceding case: it is due to the fact that a lot more harmonics are necessary to take into account the very sharp variation of the resistance in the edge of the two "doors" (infinite derivative).

\subsection{Experimental inversion}

In practice, it is very difficult to calibrate an artificial defect of known characteristics; The "double door" resistance function has been realized between two PVC square samples (same dimensions as in section 3.2) that were joined by an adhesive film that has been deposited on the whole interface but two stripes corresponding to the two "doors". The other charasteristics of this sample are: $\rho c=1.32710^{6} \mathrm{Jm}^{-3} \mathrm{~K}^{-1}, a_{x}=a_{z}=1.1310^{-7} \mathrm{~m}^{2} / \mathrm{s}$ (measured).

An infrared short wave AGEMA 782 SW Camera with a DATAMIN acquisition system was used to digitize the infrared signal (dimensional $\Delta t=0.64 \mathrm{~s}, \mathrm{~m}=100, \mathrm{~N}=120$ ). The inverted profile (obtained for an optimized value of the Laplace parameter $p$ based on a stochastic technique and simulations of inversion to minimize the estimation error - see [5]), expressed in term of 
http://dx.doi.org/10.21611/qirt.1994.038

defect thickness $e_{\mathrm{d}}\left(\right.$ with $e_{\mathrm{d}}(x)=\left(\lambda_{\mathrm{d}} / \lambda\right) e R(x), \lambda_{\mathrm{d}}=\lambda_{\text {air }}=0.026$ S.I., $p=9$ and $\left.\mathrm{n}=30\right)$ is shown in figure $3 b$ and compared with the corresponding profile measured in an optical way (first door: thickness $=0.144 \mathrm{~mm}$ for a width of $3 \mathrm{~mm}$; second door: thickness $=0.159 \mathrm{~mm}$ for a width of $5 \mathrm{~mm}$; distance of $8 \mathrm{~mm}$ between two doors). Agreement between the two profiles seems satisfactory, except for the edges and for the levels of the maxima corresponding to the two "doors". These results will be improved in the future using a function specification technique, the transfer function of the camera, other values of $p$ (least squares ?) and a better stimulation.

\section{Conclusion}

The method of integral Laplace and Fourier transforms has been used in order to estimate the variation of an in-plane interface thermal resistance starting from a transient surface temperature field recorded on the slab that has been stimulated by a Dirac heat pulse. The inversion algorithm is an analytical explicit one; A simulation study has shown that "smooth" interface resistance functions can be estimated with a satisfactory precision. The main causes of the estimation error can be studied using stochastic tools and experiment simulations. Inversion of an experimental "hard" interface resistance has been implemented; It gives some orientations to an improvement of the inversion algorithm that has to be made using the control of the estimation errors. Extension of this inversion technique to three-dimensional problems [3] with a two-dimensional resistance distribution $R(x, y)$ is also possible.

\section{REFERENCES}

[1] MAILLET (D.), DIDIERJEAN (S.), HOULBERT (A.S.) and DEGIOVANNI (D.). - Nondestructive transient thermal evaluation of delaminations inside a laminate: a thermal processing technique of infrared images, Proc.f Eurotherm Seminar 27, Quantitative Infrared Thermography, D. Balageas et al. ed., Paris, july 7-9, 1992, Editions Européennes Thermique et Industrie, pp 212-217 and X.

[2] MAILLET (D.), HOULBERT (A.S.), DIDIEJEAN (S.), LAMINE (A.S.) and DEGIOVANNI (A.). Nondestructive thermal evaluation of delaminations inside a laminate - Part I: Identification using the measurement of a thermal contrast, and Part II: The experimental Laplace transforms method, Composites Science and Technology, Elsevier Applied Science, 47, 2, pp 137-172, 1993.

[3] BATSALE (J.C.), BENDADA (A.), MAILLET (D.) and DEGIOVANNI (A.). - Distribution of a thermal contact resistance: Inversion using Laplace and Fourier Transformations and an asymptotic expansion, Inverse Problems in Engineering: Theory and Practice, Proceedings of the First International Conference. on Inverse Problems in Engineering, Palm Coast, Florida, June 13-18, 1993, editors: N. Zabaras, K. Woodbury et M. Raynaud, ASME, New York, pp 139-146,1993.

[4] BATSALE (J.C.), MAILLET (D.) and DEGIOVANNI (A.). - Extension de la méthode des quadripóles thermiques à l'aide de transformations intégrales - Calcul d'un transfert thermique au travers d'un défaut plan bidimensionnel - Int. J. Heat MassTransf., 37, 1, pp 111-127, 1994.

[5] BENDADA (A). - Tomographie infrarouge stimulée: application à des résistances d'interface. Thèse de Doctorat de I'INPL. Nancy, dec. 1994.

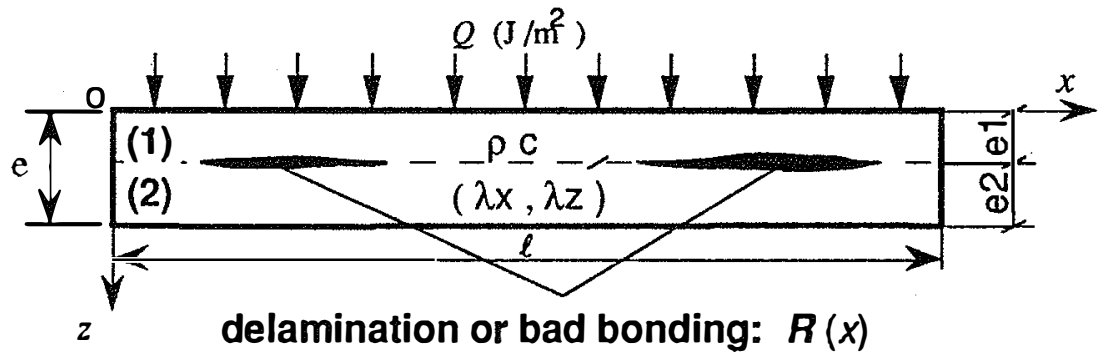

Fig. 1. - Geometry of the problem 
http://dx.doi.org/10.21611/qirt.1994.038
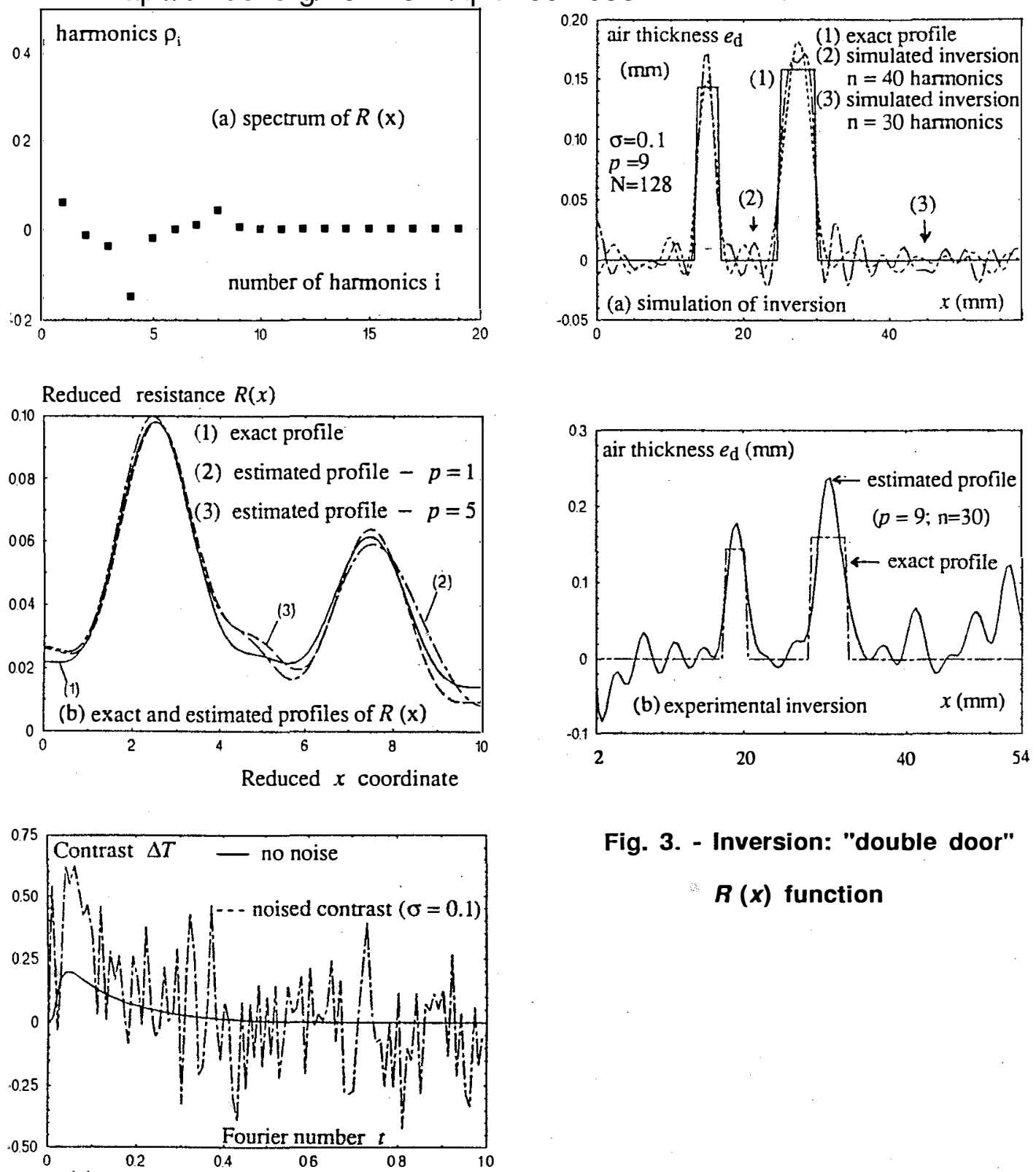

Fig. 3. - Inversion: "double door" $\boldsymbol{R}(\boldsymbol{x})$ function

(c) contrast thermog am at the location of the lower hump

Fig. 2. - Simulation of inversion: "camel-type" $R(x)$ function 